\title{
Pelvic Insufficiency Fractures after Chemoradiation for Gynecologic Malignancies: A Review of Seven Cases
}

\author{
Emeline M. Aviki ${ }^{1 *}$, Sophie M. Cowan ${ }^{2}$, Laura Young ${ }^{1}$, Marcela G. Del Carmen ${ }^{1}$, \\ Whitfield B. Growdon ${ }^{1}$, Anthony H. Russell ${ }^{3}$, Annekathryn Goodman ${ }^{1}$

\begin{abstract}
${ }^{1}$ Department of Obstetrics and Gynecology, Massachusetts General Hospital, Boston, USA; ${ }^{2}$ Department of Radiology, Massachusetts General Hospital, Boston, USA; ${ }^{3}$ Department of Radiation Oncology, Massachusetts General Hospital, Boston, USA.

Email: "eaviki@partners.org
\end{abstract}

Received October $30^{\text {th }}, 2013$; revised November $29^{\text {th }}, 2013$; accepted December $15^{\text {th }}, 2013$

Copyright (C) 2013 Emeline M. Aviki et al. This is an open access article distributed under the Creative Commons Attribution License, which permits unrestricted use, distribution, and reproduction in any medium, provided the original work is properly cited. In accordance of the Creative Commons Attribution License all Copyrights (C) 2013 are reserved for SCIRP and the owner of the intellectual property Emeline M. Aviki et al. All Copyright (C) 2013 are guarded by law and by SCIRP as a guardian.

\begin{abstract}
Background: Radiation-induced pelvic insufficiency fracture (PIF) is an important complication associated with pelvic radiation therapy (RT) for patients with gynecologic malignancies. Despite known risk factors and recent reports describing the incidence on the order of 30 percent, there has been a dearth of translational research or consensus statements to guide clinical management. Objective: The aim of this study is to describe seven cases of PIF diagnosed and managed at the Massachusetts General Hospital during a 5-year period and to perform a focused review of the literature to inform several clinical questions that remain unanswered. A secondary aim of this study is to highlight the need for additional research related to screening, prophylaxis, diagnostics, and treatment of PIF in patients with gynecologic malignancy. Methods: In the current retrospective review, we report 10 cases of PIF diagnosed over a 5-year period in 7 patients with vulvar (4), vaginal (2), and cervical (1) cancer following chemoradiation therapy at a single institution. Data were collected from the medical records by a single investigator and all diagnostic imaging was reviewed by a single radiologist to confirm the presence or absence of PIF. Results: All 7 patients were post-menopausal and received concurrent chemoradiation, 3 were over the age of 65 years old $(42.8 \%), 3$ had BMI $<25 \mathrm{~kg} / \mathrm{m}^{2}(42.8 \%), 2 \mathrm{had}$ a history of osteoporosis $(28.6 \%)$, and 1 had a history of hormone replacement therapy use (14.3\%). No patients underwent standard screening for PIF and no patients were started on prophylaxis prior to diagnosis. The plain film was the most common initial imaging performed while MRI was the most common overall study used to diagnose PIF. Median time to the development of fracture was 16 months (range 4 - 114) with femoral neck fracture being the most common (40\%) and sacral fractures trailing close behind (30\%). 7 of 10 fractures were initially managed expectantly with 1 ultimately failing expectant management and requiring surgical intervention. 4 of 10 fractures required surgical intervention. All patients had resolution of symptoms by 12 months after diagnosis. Conclusion: Radiation-induced PIF remains an important complication associated with pelvic RT. Significant risk factors have been identified and studies have compared various diagnostic imaging modalities. Future studies are needed to compare screening algorithms and evaluate the comparative effectiveness of prophylactic pharmacotherapies. Future studies are also needed to determine the cost-effectiveness of PET/CT versus MRI and compare the morbidity associated with expectant management versus surgical intervention in patients with symptomatic fractures.
\end{abstract}

Keywords: Pelvic Insufficiency Fracture; Vulva Cancer; Cervical Cancer; Radiation; Chemotherapy; Diagnostic Imaging; Fracture Screening; Fracture Prophylaxis

\section{Introduction}

Insufficiency fractures (IFs) are a subtype of stress fractures that occur when normal stress is applied to the bone with decreased mineralization and decreased elastic re-

${ }^{*}$ Corresponding author. sistance. Demineralization and reduced elastic resistance often result from osteoporosis, prior radiation therapy (RT), and prolonged corticosteroid use [1]. These factors, along with advanced age, low body weight, postmenopausal status, and others, have been reported as significant risk factors for developing pelvic insufficiency 
fractures (PIF) [2-5]. Although PIF was once thought to be a rare complication of RT, recent studies conducted in gynecologic cancer patients have reported the incidence as high as $11.1 \%-36.9 \%$ at two years and $8.2 \%-19.7 \%$ at 5 years after completion of RT [3-9]. More remote studies conducted in the mid-1990's reported incidences of PIF that range between $2.7 \%$ and $89 \%[2,10]$. Despite awareness that PIF is more common than previously expected, there continues to be an absence of standardized guidelines for screening and management of this complication.

In this case series, we report on the experience at the Massachusetts General Hospital (MGH) and present evidence from the literature with a focus on answering the following clinically-relevant questions:

- What is the optimal method of surveillance for patients after completion of RT?

- Who is at increased risk of developing PIF and how can fracture risk be reduced in these patients?

- What diagnostic imaging should be ordered for high risk patients presenting acute pelvic or hip pain?

- In cases where PIF is diagnosed, what are the different treatment options?

\section{Materials and Methods}

From November 19, 2007 through November 3, 2012, thirteen women with vulva cancer and forty-two women with cervical cancer received definitive radiotherapy with concurrent chemotherapy at Massachusetts General Hospital. During this time interval, seven patients with pelvic insufficiency fractures following chemoradiation were identified. Medical records were reviewed and patient characteristics such as age, BMI, medical comorbidities, current medications, smoking status, menopausal status, primary cancer and stage were obtained. The patient's cancer treatment history including surgery, chemotherapy, and RT were also collected. Hospital and clinic records were reviewed and presenting symptoms, date of symptom onset, imaging studies ordered, date of diagnosis, treatment offered, treatment received, and symptom control were recorded. All imaging studies including plain radiographs (plain film), computed tomography (CT), magnetic resonance imaging (MRI), and bone scintigraphy (bone scan) were performed at a single institution and reviewed by the same radiologist from the Department of Radiology who documented the presence and location of PIF in each study.

\subsection{Follow-Up}

Patient follow-up information was available for all patients in this series. Patients were evaluated post-RT at 3to 12-month intervals for assessment of disease status as well as treatment-related toxicities and complications.
This evaluation included a review of systems, physical examination, and imaging to assess response to therapy. The range of follow-up for patients was 19 to 124 months after completion of RT. Following diagnosis of PIF patients were evaluated at 2-week to 3-month intervals for assessment of symptoms and response to treatment. The range of follow-up for patients after diagnosis of PIF was 10 to 65 months.

\subsection{Diagnostic Criteria for PIF}

PIF was defined as evidence of fracture on plain film, CT, MRI or bone scan occurring within the field of irradiation. On plain films, insufficiency fractures (IF) are generally classified into two broad categories based on appearance: occult or aggressive. Occult IFs usually appear as vertical sclerotic bands, cortical disruptions, or fracture lines and are most commonly seen in the sacrum, the supra-acetabulum or the ilium. Aggressive appearing fractures resemble malignant neoplasms with findings that include areas of sclerosis and periosteal reaction. These are typically found in the parasymphysis and the pubic rami. Whether occult or aggressive in radiographic appearance, further imaging is required for a correct diagnosis of PIFs to be made [11]. On CT, findings suggestive of IF include areas of linear sclerosis or fracture lines. On MRI, findings suggestive of IF include both marrow edema and a fracture line. Marrow edema is seen as areas of increased signal intensity on $\mathrm{T} 2$-weighted images and as a hypointense linear bands on T1weighted images. A hypointense fracture line is usually evident within the area of edema on both $\mathrm{T} 1$ and $\mathrm{T} 2$ weighted images, though it is not seen in around $7 \%$ of cases. MRI can usually differentiate marrow edema secondary to IFs from malignancy, with fat-saturation and postgadolinium imaging being particularly useful in this regard [12]. On bone scan, findings suggestive of insufficiency fracture include various patterns of increased radiotracer uptake on delayed images and positive blood pool in acute cases. In the sacrum, increased uptake is seen in a vertical pattern through the sacral ala with or without a horizontal component through the sacrum. The "Honda" sign or H-pattern is considered diagnostic of sacral IFs in the correct clinical setting [12]. In vertebral bodies, findings include linear uptake in the superior endplate progressing to complete vertebral body involvement. In the proximal femur, findings include increased linear transverse uptake, with the femoral neck being the most common site. All imaging studies obtained on the seven cases were reviewed by the same radiologist from the Department of Radiology who determined whether PIF was evident based on the described, well-established, modality-specific criteria for diagnosis. 


\subsection{Endpoints and Analysis}

The time of PIF diagnosis was defined as the date of detection on imaging study. In patients with multiple fractures, the date of diagnosis for each fracture was recorded separately. Time from completion of RT to diagnosis of PIF was defined as the number of months from completion of radiation therapy to the diagnosis of PIF on imaging. Time from symptom onset to diagnosis of PIF on imaging was defined as the number of days between documentation of initial symptom in the medical record to the date of diagnosis for each PIF.

Institutional Review Board approval was obtained under protocol number 2010P002350, approved on October $15,2012$.

\section{Results}

\subsection{Patient Characteristics}

Table 1 provides a summary of the patient characteristics for the 7 patients in this series. Three different gynecologic malignancies were treated: vulvar (4), vaginal (2), cervical (1). The patients ranged in age from 46 to 86 years old and in BMI from 20 to $31.7 \mathrm{~kg} / \mathrm{m}^{2}$. All seven women were post-menopausal. Two had a history of tobacco abuse, although only one remained a smoker throughout treatment. Three of the patients had a history of hypertension; only one was actively on antihypertensive medications. One patient had diabetes and hypothyroidism, both of which were well controlled. One patient had a pre-existing diagnosis of severe osteoporosis diagnosed in her 40's with two prior known fractures in the left upper extremity; she already completed five years of estrogen replacement therapy prior to initiating RT.

\subsection{Cancer Treatment}

Table 2 includes a summary of the radiation and chemotherapy received by the 7 women in this series. All 7 women received some form of both chemotherapy and radiation therapy. Five of the 7 women received cisplatin; 3 received cisplatin with 5-FU and 1 with Xeloda. One of the 7 received Xeloda alone and one received 5-FU with Mitomycin. The women in the series received a median of 57.6 Gy of pelvic radiation. Radiation was administered with a pelvic boost for 4 of 7 women with a median boost of 13 Gy. All patients received 40 - 45 Gy of radiotherapy to future sites of PIF. Six of the seven women underwent surgery for their cancer and 3 of the 6 underwent a pelvic lymph node dissection at the time of surgery.

\subsection{Pelvic Insufficiency Fractures}

Table 3 provides a summary of information including presentation, diagnostics, treatment, and outcome associ-
Table 1. Summary of patient characteristics $(\mathbf{N}=7)$.

\begin{tabular}{ccc}
\hline Variable & Value & Range or Percent \\
\hline Age at XRT (years) & Median 64 & $46-86$ \\
BMI $\left(\mathrm{kg} / \mathrm{m}^{2}\right)$ & Median 26.6 & $21.5-31.7$ \\
Post-menopausal & $7 / 7$ & $100 \%$ \\
Past Medical History & \\
Hypertension & $3 / 7$ & $42.8 \%$ \\
Diabetes mellitus & $1 / 7$ & $14.3 \%$ \\
Osteoporosis & $2 / 7$ & $28.6 \%$ \\
Tobacco abuse & $2 / 7$ & $28.6 \%$ \\
Primary Cancer & & \\
Vulva & $4 / 7$ & $57.1 \%$ \\
Vaginal & $2 / 7$ & $28.6 \%$ \\
Cervical & $1 / 7$ & $14.3 \%$ \\
FIGO Stage & & \\
II & $2 / 7$ & $28.6 \%$ \\
III & $4 / 7$ & $57.1 \%$ \\
IV & $1 / 7$ & $14.3 \%$ \\
\hline
\end{tabular}

Table 2. Cancer treatment history $(\mathrm{N}=7)$.

\begin{tabular}{ccc}
\hline Variable & Value & Range or Percent \\
\hline Radiation Therapy & & \\
Median EBRT (Gy) & 57.6 & $44.8-68.4$ \\
Pelvic Boost & $4 / 7$ & $57.1 \%$ \\
Median Boost (Gy) & 13 & $9-23$ \\
Concurrent Chemotherapy & $7 / 7$ & $100 \%$ \\
Ciaplatin alone & $1 / 7$ & $14.3 \%$ \\
Cisplatin + 5FU & $3 / 7$ & $42.8 \%$ \\
Cisplatin + Xeloda & $1 / 7$ & $14.3 \%$ \\
5FU + Mitomycin & $1 / 7$ & $14.3 \%$ \\
Xeloda alone & $1 / 7$ & $14.3 \%$ \\
Surgical Resection & $6 / 7$ & $85.7 \%$ \\
Surgery with lymphadenectomy & $3 / 7$ & $42.8 \%$ \\
Surgery without lymphadenectomy & $3 / 7$ & $42.8 \%$ \\
\hline
\end{tabular}

ated with the ten fractures identified in this case series.

Hip pain was the most common presenting symptom associated with PIF; two patients experienced pain only with ambulation, while one experienced pain that was suprapubic in location.

Fractures were identified in five different areas of the pelvis; the most common location for PIF was on the femoral neck (40\%) with the sacrum trailing close behind $(30 \%)$. The distribution of fractures on the pelvis can be seen in Figure 1. The median time from completion of RT to diagnosis of PIF was 16 months (range $4-114$ months), with most patients developing fractures less than six months following completion of RT. When time to fracture development was broken down by fracture location, the median time for development of acetabular, sacral, and femoral neck fractures appears to be less than 
Table 3. Clinical characteristics of cases diagnosed with PIF following chemo-radiation therapy for gynecologic malignancy.

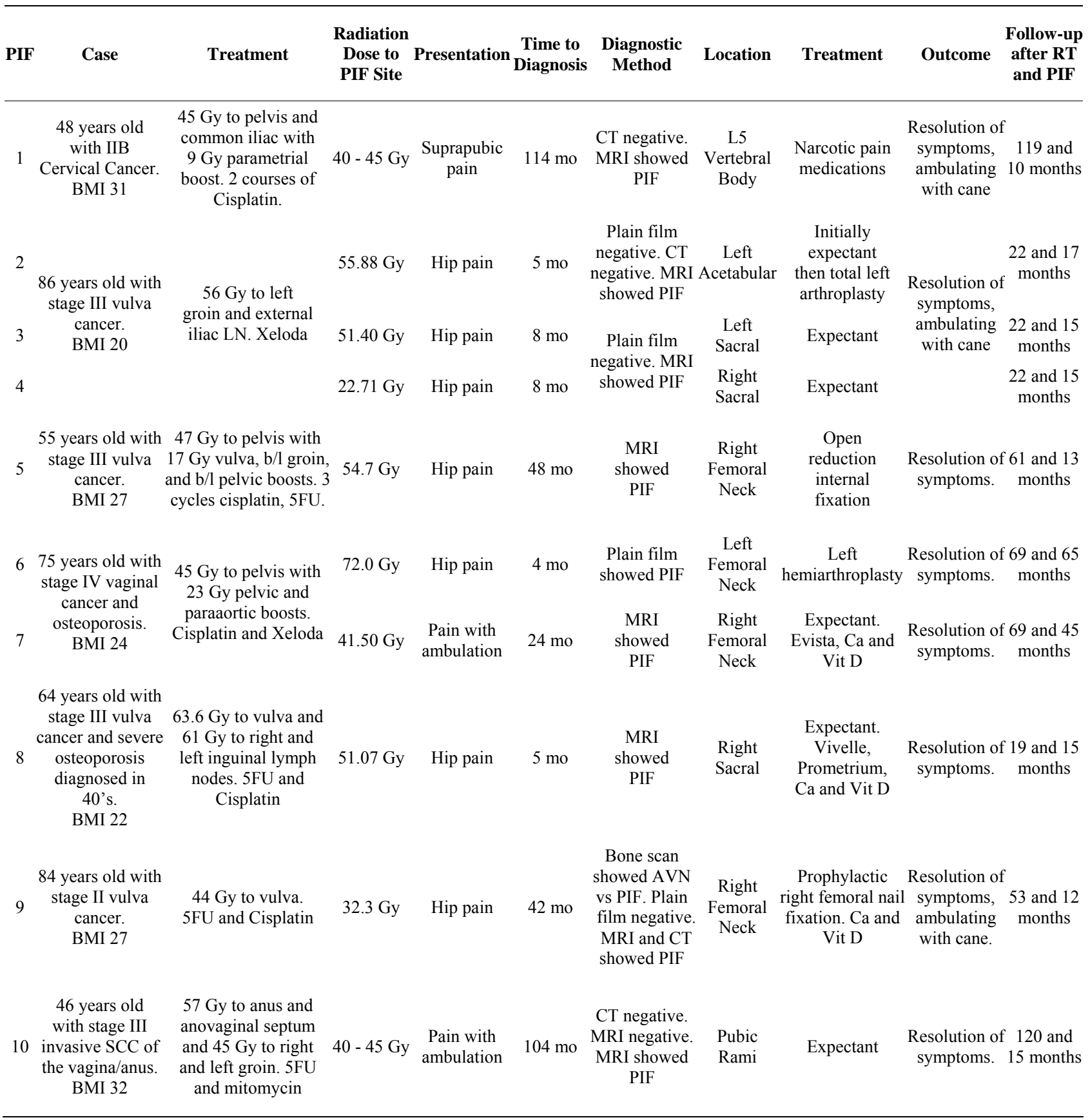

that associated with development of fractures in the vertebral bodies and the public rami (6.9 months vs 109 months, respectively). The median time for development of fractures affecting the femoral neck was 33 months (range 4 - 48), while that for vertebral body fracture was considerably longer at 114 months. Details on time to development of PIF by location can be found in Table 4 .

While a majority of the patients in the current study only developed one single PIF (71.4\%), two patients developed multiple fractures. One patient developed two separate fractures affecting the right and left femoral neck and one patient developed three separate fractures affecting the right and left sacrum as well as the left acetabulum.

\subsection{Expectant Management}

In terms of treatment, in the absence of current or impending displacement, fractures were typically treated expectantly with narcotic pain medications, NSAIDs, limitations on weight bearing and some form of physical 


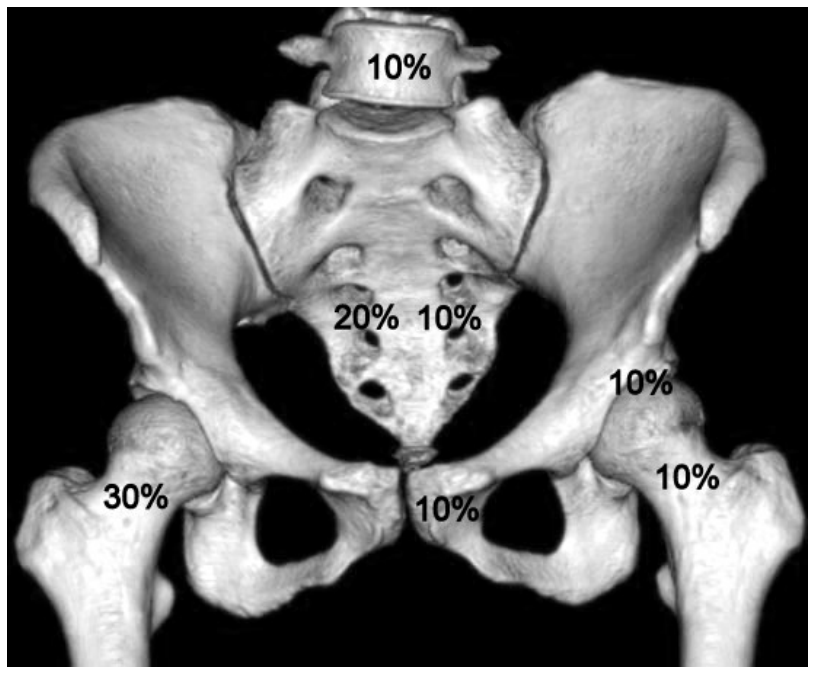

Figure 1. Distribution of ten pelvic insufficiency fractures found in seven patients $(\mathrm{N}=10)$.

Table 4. Pelvic insufficiency fracture summary.

\begin{tabular}{ccc}
\hline Location (N) & $\begin{array}{c}\text { Median Time to } \\
\text { Fracture (months) }\end{array}$ & Range (months) \\
\hline All Fractures (10) & $\mathbf{1 6}$ & $\mathbf{4 - 1 1 4}$ \\
Femoral Neck (4) & 33 & $4-48$ \\
Sacrum (3) & 5 & $5-8$ \\
Acetabulum (1) & 5 & N/A \\
Pubic Rami (1) & 104 & N/A \\
L5 Vertebrae (1) & 114 & N/A \\
\hline
\end{tabular}

therapy.

There were a total of seven fractures in four different patients that were initially treated with expectant management. One of the seven fractures ultimately failed expectant management and required surgical treatment. This case was that of a fracture located in the left acetabulum which continued to cause pain and progressed despite expectant measures. It ultimately developed a protrusion that required complex total hip arthroplasty. In the remaining six fractures in four different patients, expectant management resulted in resolution of symptoms and improved functional capacity.

In the series of cases that underwent expectant management, the median follow-up after diagnosis of PIF was 15 months (range 10 - 45 months). At time of last patient follow-up, symptoms had resolved for all 6 expectantly managed fractures.

\subsection{Surgical Management}

Four out of seven patients in this series underwent surgical management for four different fractures. Treatments included open reduction with internal fixation, prophylactic internal fixation, hemiarthroplasty, and total complex arthroplasty.
One patient was diagnosed with a right femoral neck fracture 42 months following completion of radiation therapy and received an elective prophylactic right femoral nail fixation in the setting of advanced age of 88 and severe osteopenia throughout the pelvis. At 4 month post-op follow-up, the patient's symptoms persisted and she continued to require narcotic medications for pain control; however, by 12 months, her symptoms had completely resolved and she was able to ambulate without assistance.

Among the three non-elective surgical cases, there was only one case where expectant management was attempted for three co-existing fractures, yet failed due to persistence of symptoms and progression of the fracture in left acetabulum. In this case, the left acetabular fracture progressed to the point of protrusion which ultimately required a complex left total hip arthroplasty.

One patient developed a left femoral neck fracture 4 months following completion of RT which was treated with a left hemiarthroplasty; this patient then went on to develop a right femoral neck fracture 24 months following completion of RT, which was managed successfully with expectant management including initiation of Evista along with calcium and vitamin D supplementation.

The final surgical case experienced an acute onset of hip pain one day after a normal routine bone scan and on subsequent imaging was found to have a right femoral neck fracture with high suspicion for hematoma formation which was treated with an open reduction with internal fixation two days later.

Median follow-up after diagnosis of PIF for patients undergoing surgical management was 15 months (range 12 - 65 months). Non-elective surgical management in these cases resulted in resolution of symptoms and improved functional capacity. The one case managed with elective prophylactic femoral nail fixation also resulted in resolution of symptoms and improved functional capacity by 12 month follow-up.

\subsection{Diagnostic Imaging}

The most common initial test ordered when a patient in the current study presented with new onset hip pain was the plain film. Though not the most common initial test, MRI was the most common study ordered during the work-up of new onset hip or pelvic pain. Furthermore, MRI was required for diagnosis of PIF in all but one case, where plain film alone was sufficient to diagnose a complete left femoral neck fracture.

In this series, when plain film was the initial imaging study, an average of 2 studies (range 1 - 3) were required to reach a diagnosis of PIF. When MRI was the initial imaging study $(\mathrm{n}=3)$, no additional imaging was needed to reach a diagnosis of PIF. In the one case where bone scan was the initial imaging study ordered, an abnormal- 
ity was confirmed on bone scan; however, additional imaging was required to determine what the abnormality represented. In this case the bone scan could not differentiate between AVN versus PIF so a plain film was ordered and returned negative, followed by CT and MRI which both confirmed that a PIF was present. Details regarding the sequence of imaging studies ordered in each case can be found in Table 5. Select cases of insufficiency fracture diagnosed using multiple imaging modalities are illustrated in Figures 2-4.

\section{Discussion}

Over the five-year study period, 7 cases of pelvic insufficiency fracture were diagnosed in women who had undergone radiation therapy for gynecologic malignancies at Massachusetts General Hospital. All 7 patients were post-menopausal and received concurrent chemoradiation, 3 were over the age of 65 years old (42.8\%), 3 had BMI less than $25 \mathrm{~kg} / \mathrm{m}^{=}(42.8 \%), 2$ had a history of osteoporosis $(28.6 \%)$, and 1 had a history of hormone replacement therapy use (14.3\%). All fractures occurred within the irradiated field and were at locations that received significant doses of radiation (40 - 45 Gy). No patients underwent standard screening for PIF and no patients were started on prophylaxis prior to diagnosis. Plain film was the most common initial imaging performed while MRI was the most common overall study used to diagnose PIF. Median time to development of fracture was 16 months (range 4 - 114) with femoral neck fracture being the most common $(40 \%)$ and sacral fractures trailing close behind (30\%). 7 of 10 fractures were initially managed expectantly with 1 ultimately failing expectant management and requiring surgical intervention. 4 of 10 fractures required surgical intervention. All patients had resolution of symptoms by 12 months after diagnosis. The MGH experience exposes a significant need for future research to inform evidence based clinical management of PIF in patients with gynecologic malignancies undergoing pelvic radiation therapy.

\subsection{What Is the Optimal Method of Surveillance for Patients after Completion of RT?}

In women with gynecologic malignancy who have completed pelvic RT, no formal or suggested screening algorithms have been published to guide clinicians on PIF surveillance. On the topic of surveillance, there are three specific questions that will be important to address:

1) When should surveillance begin and how long should it last?

2) What should surveillance involve?

3) How long after completion of RT should surveillance continue?

At MGH, after completion of RT, patients are seen at
3 - 4 months intervals for the first 2 years and every six months thereafter, with routine imaging every $4-6$ months. The focus of these visits is to assess for chemoradiation related toxicity and cancer progression. There is no formal surveillance in place to screen for PIF in these patients. Since there are no published studies evaluating the topic of surveillance in these patients, findings in the existing literature can be used to guide our approach to surveillance.

Evidence from retrospective studies suggest that the median time from completion of RT to development of PIF can range anywhere from 6 to 20 months [4-6,8,9, 13,14]. This is consistent with the institutional experience at MGH where the data shows a median of 16 months for all fractures and 6.5 months if the observational period is limited to 5 years, which was the maximum period observed in the reviewed studies.

There are additional insights offered in two prospective studies where routine surveillance was conducted using imaging studies. In 2012 Tokomaru et al. published a prospective multi-institutional study on 59 cervical cancer patients who underwent definitive pelvic RT. In this study, patients were evaluated by both pelvic CT and MRI at 3, 6, 12, 18, and 24 months after completion of RT [3]. The cumulative incidence of IF was $36.9 \%$ at 2 years in all patients and $16.1 \%$ in symptomatic patients [3]. In 1996, Blomie et al. published a prospective study conducted on 18 women who underwent definitive RT for advanced cervical cancer [2]. These patients were assessed using MRI before, during, and after RT. After RT, MRI was performed at 3-month intervals for the first year then 6-month intervals until 30 months. The cumulative incidence of PIF was $89 \%$ during the 30 month study period. Focal lesions were not seen until 7 weeks post-RT with the greatest number of lesions developing between 3 and 18 months following completion of RT. Signal changes on MRI could be seen in the pelvic bones

Table 5. Diagnostic imaging used to diagnose PIF.

\begin{tabular}{ccccc}
\hline $\boldsymbol{P I F}$ & Imaging \#1 & Imaging \#2 & Imaging \#3 & Time to diagnosis \\
\hline 1 & CT (-) & MRI (+) & & 4 days \\
2 & Plain film (-) & CT (-) & MRI (+) & 5 days \\
3 & Plain film (-) & MRI (+) & & 11 days \\
4 & Plain flim (-) & MRI (+) & 11 days \\
5 & MRI (+) & & & 2 days \\
6 & Plain flim (+) & & 4 days \\
7 & MRI (+) & & MRI (+) and & days \\
8 & MRI (+) & & CT (+) & N/A \\
9 & Bone scan & Plain film (-) & MRI (+) & 21 days \\
10 & C?AVN vs PIF) & & MRI (-) & MVT
\end{tabular}



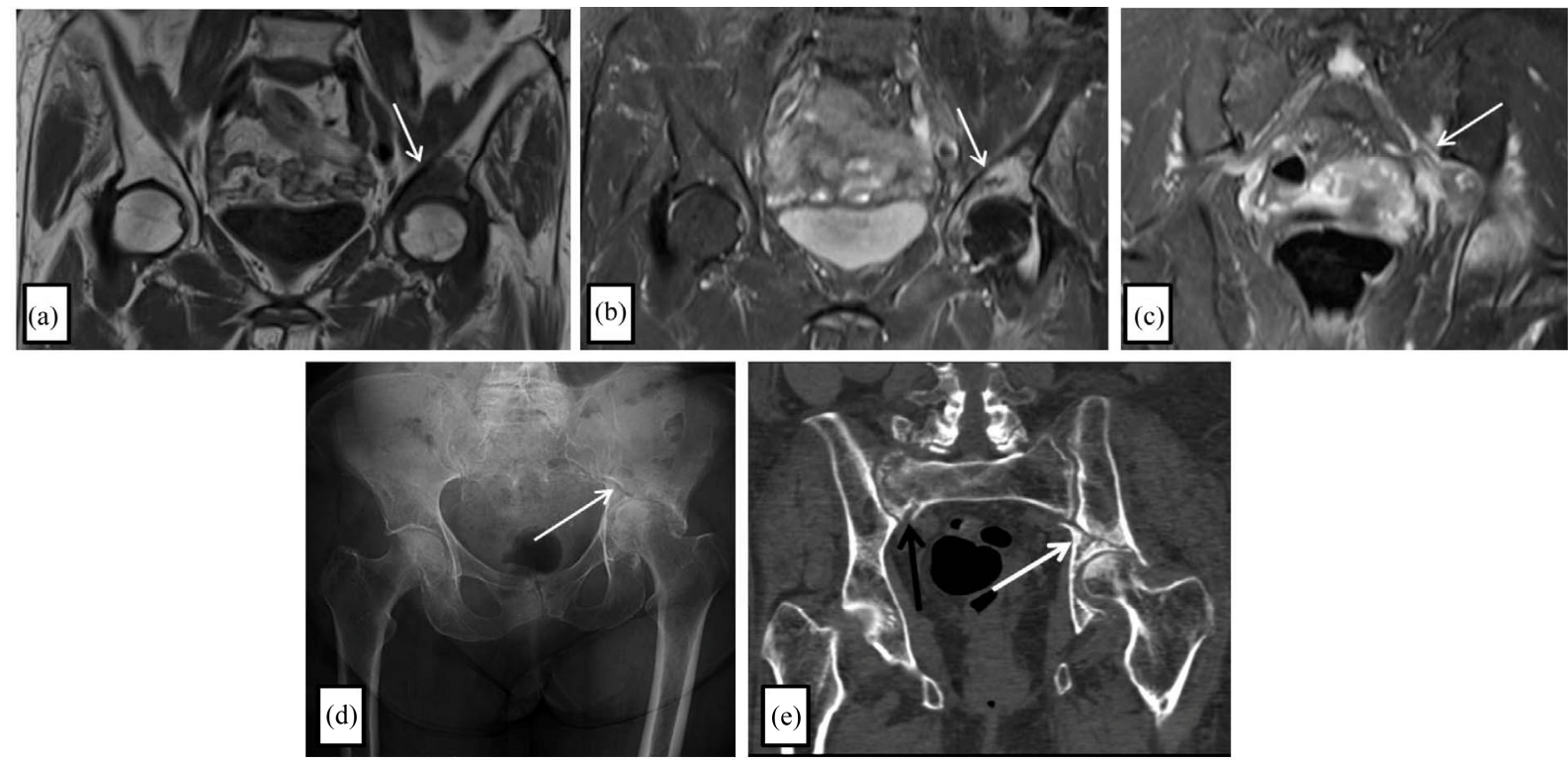

Figure 2. Despite negative plain film and CT, T1 (a) and T2 (b) weighted coronal MRI images demonstrate curvilinear hypointensity in the left superior acetabular region surrounded by bone marrow edema consistent with insufficiency fracture. T2 (c) weighted coronal MRI image demonstrates signal abnormality in the left inferior sacral ala that likely represents a developing insufficiency fracture. 5 months later, repeat plain film (d) and coronal CT (e) demonstrate a subacute fracture of the left superior acetabulum with surrounding sclerosis (white arrows). A comminuted right sacral fracture is also present on CT that is not well visualized on the radiograph (black arrow).
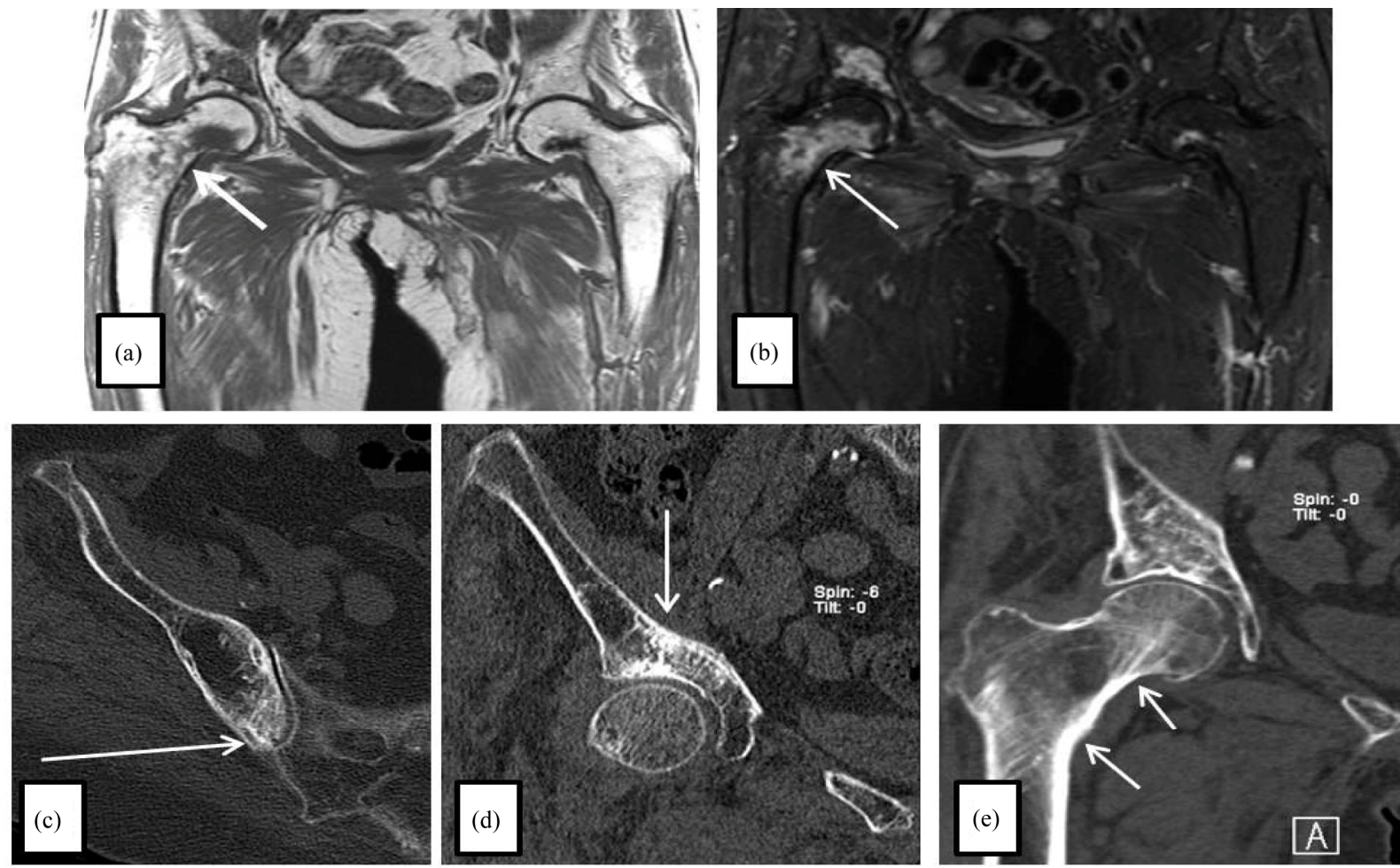

Figure 3. Right femoral neck fracture is seen on Coronal T1 (a) and T2 (b) weighted MRI images (white arrows) which demonstrate signal abnormality and surrounding $\mathrm{T} 2$ hyperintense bone marrow edema in the right femoral neck and intertrochanteric region concerning for an insufficiency fracture. Findings are more subtle on axial (c) and coronal (d, e) CT images which demonstrate subtle increased sclerosis in the posterior medial iliac bone adjacent to the sacroiliac joint (c), the supraacetabular right ilium (d), and the right femoral neck (e). No displaced fracture line is visualized; however, these findings, in conjunction with the previously seen abnormal signal on MRI, likely represent healing insufficiency fractures. 

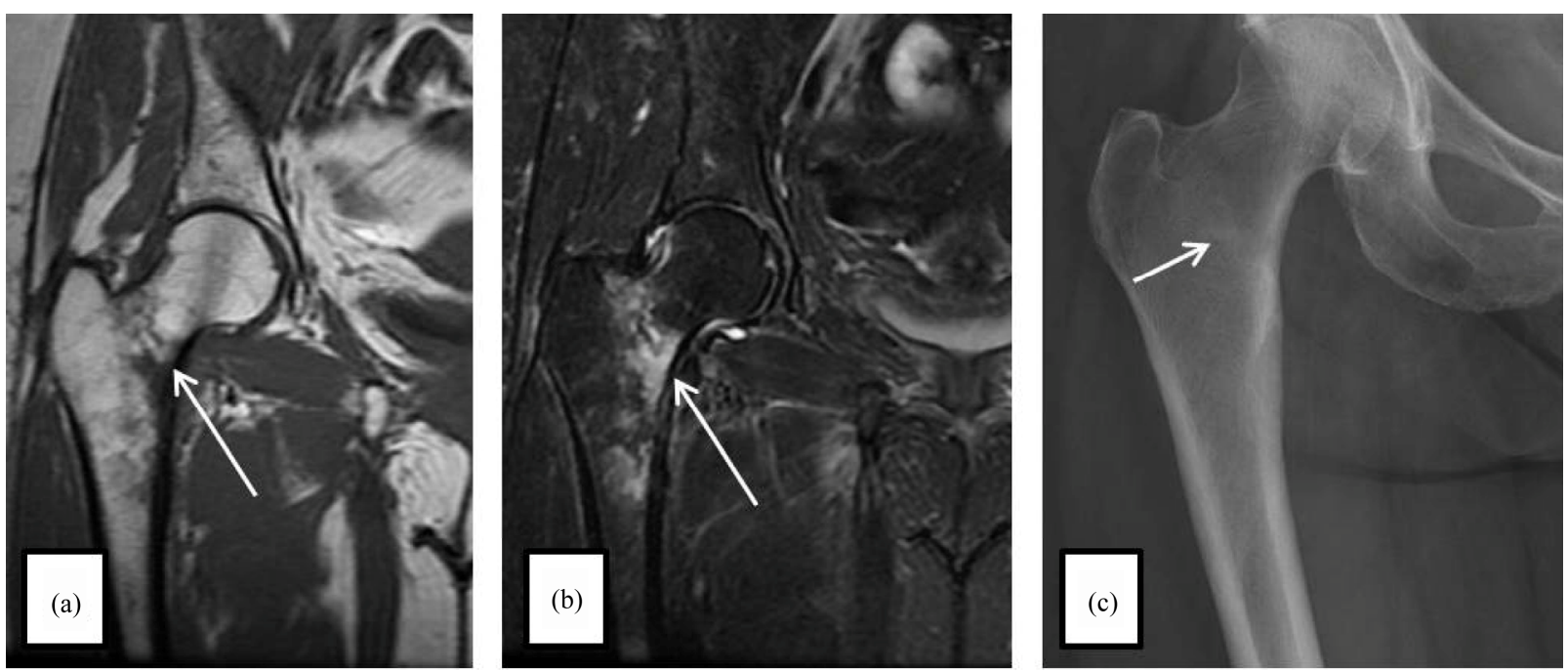

Figure 4. T1 (a) weighted coronal MRI image demonstrates linear hypointensity in the right femoral neck and T2 (b) weighted MRI image demonstrates surrounding hyperintense bone marrow edema. These findings are consistent with an insufficiency fracture. Subsequent plain film (c) of the right hip shows only linear sclerosis in the intertrochanteric region of the right proximal femur.

until 24 months after completion of RT. Only 10 out of the 18 patients complained of pelvic pain, and those with pain had a mean of 4 lesions with 1 or more that were larger than $1 \mathrm{~cm}^{2}$, whereas all lesions in patients without pain were less than $1 \mathrm{~cm}^{2}$. In both of these studies, all patients with symptomatic fractures experienced complete resolution of symptoms by the end of the observational period.

In another study that addresses a possible preferred modality for screening, Park et al. reports on 117 patients who underwent first PET/CT within 1 year after radiotherapy for cervical cancer compared to 118 patients who received $\mathrm{CT}$ alone, unless they had a specific symptoms like hip pain which would warrant an additional study such as MRI or bone scan. In the patients who underwent $\mathrm{PET} / \mathrm{CT}$ within one year, the detection rate for PIF was $16.1 \%$ whereas in the non-PET/CT group the detection rate was only $3.8 \%$ [15].

From retrospective data, we learn that a significant number of fractures develop within the first year after completion of RT, making screening during this period the most critical. From the prospective literature, we learn that radiation induced fractures do not typically occur earlier than 2 months following completion of RT, that many occur in the absence of symptoms, and that patients with symptoms are more likely to have multiple and more significant fractures. Lastly, from the Park study, we gain insight into PET/CT and its possible value given its superiority to $\mathrm{CT}$ alone coupled by its routine use at most institutions for cancer surveillance.

Based on these findings, future research is needed to determine the cost/benefit associated with initial screening starting at 3,6 , or 12 months as well as cost-benefit of using different imaging modalities for screening. Research is also needed to determine the cost-benefit to screening beyond 2 - 3 years. Lastly, if routine screening is to be adopted, there will need to be evidence that early diagnosis, particularly in asymptomatic patients, leads to improved patient outcomes, namely reduction of morbidity and mortality from fractures, particularly in asymptomatic patients.

\subsection{Who Is at Increased Risk of Developing PIF and How Can Fracture Risk Be Reduced in These Patients?}

There are many risk factors for development of radiation-induced PIF that have been identified in the literature. While risks factors for PIF have been identified, there is limited evidence to guide use of prophylaxis in high risk patients.

Steroid use has been shown to be associated with osteoporosis and increased fracture risk [1]. Chemotherapy used concurrently with pelvic radiotherapy is known to increase radiation toxicity, including bone toxicity which has been thought to predispose to PIF; however, studies have reported mixed results on this topic [16]. Steroids are often used during chemotherapy, which may be responsible for the increased risk associated with concurrent chemotherapy found in some studies [17]. Other studies, however, have not found concurrent chemotherapy to be a significant risk factor for radiation induced PIF $[6,8,9,14]$.

Many studies have found osteoporosis to be a signifycant risk factor for radiation-induced PIF [14,15,17]. There are two mechanism considered for the development of radiation induced fractures in the setting of os- 
teoporosis: the direct effect of irradiation on in-field bone marrow density (BMD) and the secondary effect on systemic BMD via decreased levels of estradiol [18]. Many studies have also found old age $[3,5,8,15]$, low body weight $[3,4,6,8,14,15]$, postmenopausal status $[2,5]$, and history of Hormone Replacement Therapy (HRT) [14] to be significant risk factors for radiation-induced PIFs. A list of risk-factors for PIF can be found in Table 6.

There may be a correlation between radiation intensity and development of PIF [8]. In a study of 557 cervical cancer patients who received whole-pelvic RT, radiation dose greater than 50.4 Gy and RT with curative intent were found to be significant risk factors for PIF [8]. In more recent studies, however, these factors have not demonstrated a significantly impact on the rate of PIF $[3,15]$. In one of these studies, conducted by Tokumaru et al., all $44 \mathrm{IF}$ sites were estimated to have a median radiation dose of 49 Gy with 38 sites estimated at doses greater than $45 \mathrm{~Gy}$, which the authors suggest raises the possibility of a threshold dose for IF at approximately 45 Gy. In the current series, 6 of the 7 cases received a cumulative pelvic RT dose of greater than $50.4 \mathrm{~Gy}$; how-

Table 6. Significant risk factors for pelvic insufficiency fracture.

\begin{tabular}{|c|c|c|}
\hline Risk Factor & Details & $\begin{array}{l}\text { Corresponding } \\
\text { Study }\end{array}$ \\
\hline Corticosteroid Use & Significant Risk Factor & {$[1]$} \\
\hline \multirow{2}{*}{$\begin{array}{c}\text { Concurrent } \\
\text { Chemotherapy }\end{array}$} & $\begin{array}{c}\text { No Significant Relationship } \\
\text { Found in Pelvic Cancer } \\
\text { Patients }\end{array}$ & {$[6,8,9,14]$} \\
\hline & $\begin{array}{c}\text { Significant Relationship } \\
\text { Found only in Rectal Cancer } \\
\text { Patients }\end{array}$ & [17] \\
\hline \multirow[t]{2}{*}{ Osteoporosis } & Significant Risk Factor & {$[14,15,17]$} \\
\hline & $\geq 55$ years & {$[8]$} \\
\hline \multirow[t]{7}{*}{ Old Age } & $\begin{array}{l}\text { Mean age } 69 \text { years in the } \\
\text { PIF groups and } 59 \text { years in } \\
\text { the unaffected group }\end{array}$ & {$[5]$} \\
\hline & $>70$ years & {$[3]$} \\
\hline & $>75$ years & {$[15]$} \\
\hline & $\leq 49 \mathrm{~kg}$ & [4] \\
\hline & $<50 \mathrm{~kg}$ & {$[3]$} \\
\hline & $<55 \mathrm{~kg}$ & [8] \\
\hline & $<23 \mathrm{~kg} / \mathrm{m}^{2}$ & {$[15]$} \\
\hline \multirow[t]{2}{*}{ Low Body Weight } & $\begin{array}{l}\text { Median BMI } 26 \mathrm{~kg} / \mathrm{m}^{2} \text { in the } \\
\text { PIF group and } 28 \mathrm{~kg} / \mathrm{m}^{2} \text { in } \\
\text { the unaffected group }\end{array}$ & {$[6]$} \\
\hline & $\begin{array}{l}\text { Median BMI } 25.9 \mathrm{~kg} / \mathrm{m}^{2} \text { in } \\
\text { PIF group and } 27.2 \mathrm{~kg} / \mathrm{m}^{2} \text { in } \\
\text { the unaffected group }\end{array}$ & {$[14]$} \\
\hline Menopausal Status & Post-menopausal & {$[2,5]$} \\
\hline History of HRT & $\begin{array}{l}\text { History of HRT who were } \\
\text { not Currently Using }\end{array}$ & {$[14]$} \\
\hline
\end{tabular}

ever, all cases received fracture-site specific doses that ranged between 40 - $45 \mathrm{~Gy}$, which is below the previously suggested threshold. Nevertheless, all cases received significant doses to sites of PIF which was necessary due to the location of their cancers. Future studies are needed to determine whether dose-specific thresholds do in fact exist in order to factor into pre-treatment risk stratification.

In patients with known risk factors for osteoporosis or for radiation-induced PIF, potential interventions to prevent PIF should be based on standard treatments to promote bone health and may be initiated before, during or after RT.

One approach, recommended by $\mathrm{Oh}$ et al., would be to prevent PIF after RT through combined treatment of osteoporosis [8]. Osteoporosis often remains undetected in cancer patient until fracture occurs [19]. Studies have found that bone density testing is performed in only $3 \%$ $32 \%$ of high risk patient $[20,21]$. Guidelines published by the US Preventive Services Task Force recommend screening of women aged 65 years or older or in younger women with 10 -year cumulative fracture risk of $9.3 \%$ or greater, which would include all women with gynecologic malignancy receiving pelvic radiation therapy [22]. Screening should include a baseline bone marrow density (BMD) measurement with repeat testing as early as two years after a normal initial BMD.

BMD is classified according to T-score, the number of standard deviations above or below the mean BMD for healthy adults. A T-score of -2.5 or less is classified as osteoporosis; between -2.5 and -1.0 is considered low bone density (osteopenia); and -1 or greater is considered normal.

Based on BMD scores, medications to decrease fracture risk may be indicated. Based on a recent AHRQ comparative effectiveness review, there is limited evidence supporting a potential benefit of vitamin D and calcium (alone or in combination) in lowering fracture risk. With regard to other interventions, bisphosphonates, denosumab, raloxifene, and teriparatide reduce vertebral fracture risk, but only alendronate, risedronate, zoledronic acid, and denosumab have proven to reduce hip fracture risk, which is an important point to consider in patients after pelvic RT who are more likely to have PIF develop the sacrum and femoral neck than in the vertebral bodies. Raloxifene does not reduce the risk of hip or other nonvertebral fractures. Most osteoporosis intervenetions have possible adverse effects, which should be taken into account in when deciding what treatment would be best suited for PIF prophylaxis [23].

\subsection{What Diagnostic Imaging Should Be Ordered for High Risk Patients Presenting with Acute Pelvic or Hip Pain?}

Diagnosing pelvic insufficiency fractures in gynecologic 
oncology patients is often difficult due to symptoms and radiographic findings that can be mistaken for recurrent disease or bony metastasis. Consistent with findings in the current study, most cases present with pelvic, lower back, and/or hip pain, but some patients may be asymptomatic [15]. When a patient presents with these symptoms, it is important understand the limitations of each imaging modality and more importantly, to know which modality is most likely to both detect PIFs and adequately differentiate them from pathologic fractures.

Conventional radiographs are often inconclusive [11, 12] and bone scan is limited by low specificity, which requires an additional test to confirm diagnosis [11].

In a study comparing the rate of PIF detection using $\mathrm{CT}$ versus MRI, Cabarrus et al. found $\mathrm{CT}$ to be inferior to MRI. In this study of 64 subjects, CT diagnosed only $69 \%$ of fractures ( 89 of 129 ), while MRI diagnosed $99 \%$ of fractures (128 of 129) [24]. Findings in the current study are consistent with that of Cabarrus et al. When CT was used as the initial imaging study, it failed to diagnose PIF in both instances where MRI subsequently detected PIF.

In a more recent study which was previously described, Park et al. found that in 117 patients who underwent PET/CT within one year after completion of RT, $16.1 \%$ of PIF were identified. However, in 118 similar patients who received CT alone (unless they had a specific symptoms like hip pain which would warrant an additional study such as MRI or bone scan), only $3.8 \%$ of PIF were identified [15].

$\mathrm{PET} / \mathrm{CT}$ and MRI have superior detection rates compared to other modalities. Similarly, both modalities are able to distinguish between pathologic fractures and PIF. In the absence of comparative effectiveness studies, the decision to use one over the other should be based on institutional preference and availability.

\subsection{In Cases Where PIF Is Diagnosed, What Are the Different Treatment Options?}

Treatment of pelvic insufficiency fractures typically begins with conservative management, emphasizing limited weight bearing, symptomatic pain relief, and physical therapy [25-28]. When expectant management is unsuccessful in resolving symptoms, surgical interventions may be considered. In reports of patients who have refractory pain after expectant management, clinical improvements have been demonstrated after surgically invasive measures, such as prophylactic or medically indicated in situ fixation, percutaneous cement osteoplasty, and metallic stent scaffolding [26,29-33]. Moreover, conservative management has been shown to be thoroughly effective, sometimes with NSAIDs and physical therapy alone, and improvement can typically be seen within weeks after PIF is diagnosed, with resolution of symptoms by 1 - 35 months $[1,2,4,5,27,28]$. In the current study, both expectant and surgical management were employed based on the given clinical scenario. At MGH, in cases where initial expectant management is ineffective or where radiographic findings reveal progression of PIF, a multidisciplinary approach to management is taken involving the patient, the primary gynecologic oncologist, the radiation oncologist, and an experienced orthopedic surgeon. All patients in the current study, whether managed expectantly or surgically, had complete resolution of symptoms and had improved functional capacity by 12 month follow-up.

\section{Conclusion}

Radiation-induced PIFs are an important complication associated with pelvic RT. Future research is needed to inform evidence based on clinical management of PIF in patients with gynecologic malignancies undergoing pelvic radiation therapy. Key areas of opportunity identified in this review include: 1) developing optimal screening guidelines, 2) identifying high risk patients who would benefit from prophylaxis, 3) evaluating the comparative effectiveness of pharmacotherapies for prophylaxis in PIF, 4) evaluating the comparative effectiveness of PET/ CT versus MRI for screening and diagnosis, and 5) comparing the morbidity associated with expectant management versus surgical interventions for treatment of patients with symptomatic fractures.

\section{REFERENCES}

[1] S. J. Huh, "Post Pelvic Radiotherapy Bony Changes," The Journal of the Korean Society for Therapeutic Radiology and Oncology, Vol. 27, No. 1, 2009, pp. 1-9. http://dx.doi.org/10.3857/jkstro.2009.27.1.1

[2] V. Blomlie, E. K. Rofstad, K. Talle, et al., "Incidence of Radiation-Induced Insufficiency Fractures of the Female Pelvis: Evaluation with MR imaging," American Journal of Roentgenology, Vol. 167, No. 5, 1996, pp. 1205-1210. http://dx.doi.org/10.2214/ajr.167.5.8911181

[3] S. Tokumara, T. Toita, M. Oguchi, et al., "Insufficiency Fractures After Pelvic Radiation Therapy for Uterine Cervical Cancer: An Analysis of Subjects in a Prospective Multi Institutional Trial," International Journal of Radiation Oncology * Biology * Physics, Vol. 84, No. 2, 2012, pp. 195-200.

http://dx.doi.org/10.1016/j.ijrobp.2012.03.042

[4] I. Ogino, N. Okmoto, Y. Ono, et al., "Pelvic Insufficiency Fractures in Postmenopausal Women with Advanced Cervical Cancer Treated by Radiotherapy," Radiotherapy and Oncology, Vol. 68, No. 1, 2003, pp. 61-67. http://dx.doi.org/10.1016/S0167-8140(03)00128-2

[5] H. Ikushima, K. Osaki, S. Furutani, et al., "Pelvic Bone Complications Following Radiation Therapy of Gynecologic Malignancies: Clinical Evaluation of Radiation Induced Pelvic Insufficiency Fractures," Gynecologic On- 
cology, Vol. 103, No. 3, 2006, pp. 1100-1114. http://dx.doi.org/10.1016/j.ygyno.2006.06.038

[6] K. M. Schmeler, A. Jhingran, R. B. Iyer, et al., "Pelvic Fractures after Radiotherapy for Cervical Cancer: Implications for Survivors," Cancer, Vol. 116, No. 3, 2010, pp. 625-630. http://dx.doi.org/10.1002/cncr.24811

[7] N. N. Baxter, E. B. Habermann, J. E. Tepper, et al., "Risk of Pelvic Fractures in Older Women Following Pelvic Irradiation," JAMA, Vol. 294, No. 20, 2005, pp. 2587-2593. http://dx.doi.org/10.1001/jama.294.20.2587

[8] D. Oh, S. J. Huh, H. Nam, et al., "Pelvic Insufficiency Fracture after Pelvic Radiotherapy for Cervical Cancer: Analysis of Risk Factors," International Journal of Radiation Oncology * Biology * Physics, Vol. 70, No. 4, 2008, pp. 1183-1188.

http://dx.doi.org/10.1016/j.ijrobp.2007.08.005

[9] J. W. Kwon, S. J. Huh, Y. C. Yoon, S. H. Choi, J. Y. Jung, D. Oh and B. K. Choe, "Pelvic Bone Complications after Radiation Therapy of Uterine Cervical Cancer: Evaluation with MRI," American Journal of Roentgenology, Vol. 191, No. 4, 2008, pp. 987-994. http://dx.doi.org/10.2214/AJR.07.3634

[10] P. Bliss, C. Parsons and P. Blake, "Incidence and Possible Etiological Factors in the Development of Pelvic Insufficiency Fractures Following Radical Radiotherapy," British Journal of Radiology, Vol. 69, No. 822, 1996, pp. 548-554. http://dx.doi.org/10.1259/0007-1285-69-822-548

[11] W. C. Peh, P. Khong, Y. Yin, W. Y. Ho, N. S. Evans, L. A. Gilula, H. W. Yeung and A. M. Davies, "Imaging of Pelvic Insufficiency Fractures," Radiographics, Vol. 16, No. 2, 1996, pp. 335-348.

[12] E. M. Lyders, C. T. Whitlow, M. D. Baker and P. P. Morris, "Imaging and Treatment of Sacral Insufficiency Fractures," American Journal of Neuroradiology, Vol. 31, No. 1, 2010, pp. 201-210. http://dx.doi.org/10.3174/ajnr.A1666

[13] S. J. Huh, B. Kim, M. K. Kang, et al., "Pelvic Insufficiency Fractures after Pelvic Irradiation in Uterine Cervix Cancer," Gynecologic Oncology, Vol. 86, No. 3, 2002, pp. 264-268. http://dx.doi.org/10.1006/gyno.2002.6756

[14] K. K. Shih, M. R. Folkert, M A. Kollmeier, N. R. AbuRustum, Y. Sonoda, M. M. Leitao, R. R. Barakat and K. M. Alektiar, "Pelvic Insufficiency Fractures in Patients with Cervical and Endometrial Cancer Treated with Postoperative Pelvic Radiation," Gynecologic Oncology, Vol. 128, No. 3, 2013, pp. 540-543. http://dx.doi.org/10.1016/j.ygyno.2012.12.021

[15] S. Park, J. Kim, J. Lee, et al., "Pelvic Insufficiency Fracture after Radiotherapy in Patients with Cervical Cancer in the Era of PET/CT," Journal of Radiation Oncology, Vol. 29, No. 4, 2011, pp. 269-276. http://dx.doi.org/10.3857/roj.2011.29.4.269

[16] P. J. Jenkins, D. J. Montefiore and S. J. Arnott, "Hip Complications Following Chemoradiationtherapy," Vol. 7, 1995, pp. 123-126.

[17] H. J. Kim, P. J. Boland, D. S. Meredith, E. Lis, Z. Zhang, W. Sih, et al., "Fractures of the Sacrum after Chemoradiation for Rectal Carcinoma: Incidence, Risk Factors, and Radiographic Evaluation," International Journal of Radiation Oncology Biology \& Physics, Vol. 84, No. 3, 2012, pp. 649-699.

[18] N. Okonogi, J. I. Saitoh, Y. Suzuki, et al., "Changes in Bone Mineral Density in Uterine Cervical Cancer Patients after Radiation Therapy," International Journal of Radiation Oncology * Biology * Physics, Vol. 87, No. 5, 2013, pp. 968-974.

http://dx.doi.org/10.1016/j.ijrobp.2013.08.036

[19] T. A. Guise, "Bone Loss and Fracture Risk Associated with Cancer Therapy," Oncologist, Vol. 11, No. 10, 2006, pp. 1121-1131. http://dx.doi.org/10.1634/theoncologist.11-10-1121

[20] M. D. Smith, W. Ross and M. J. Ahern, "Tissing a Therapeutic Window of Opportunity: An Audit of Patients Attending a Tertiary Teaching Hospital with Potentially Osteoporotic Hip and Wrist Fractures," The Journal of Rheumatology, Vol. 28, No. 11, 2001, pp. 2504-2508.

[21] C. A. Morris, H. Cheng, D. Cabral, et al., "Predictors of Screening and Treatment of Osteoporosis: A Structured Review of the Literature," The Endocrinologist, Vol. 14, No. 2, 2004, pp. 70-75. http://dx.doi.org/10.1097/01.ten.0000123564.40707.84

[22] H. D. Nelson, E. M. Haney, T. Dana, C. Bougatsos and R. Chou, "Screening for Osteoporosis: An Update for the U. S. Preventive Services Task Force," Annals of Internal Medicine, Vol. 153, No. 2, 2010, pp. 99-111. http://dx.doi.org/10.7326/0003-4819-153-2-201007200-0 $\underline{0262}$

[23] C. J. Crandall, S. J. Newberry, A. Diamant, et al., "Treatment To Prevent Fractures in Men and Women with Low Bone Density or Osteoporosis: Update of a 2007 Report, [Internet," Comparative Effectiveness Reviews, No. 53, Agency for Healthcare Research and Quality (US), Rockville, 2012. http://www.ncbi.nlm.nih.gov/books/NBK92566/

[24] M. C. Cabarrus, A. Ambekar, Y. Lu, et al., "MRI and CT of Insufficiency Fractures of the Pelvis and the Proximal Femur," American Journal of Roentgenology, Vol. 19, No. 4, 2008, pp. 995-1001. http://dx.doi.org/10.2214/AJR.07.3714

[25] P. Tai, A. Hammond, J. Van Dyk, et al., "Pelvic Fractures Following Irradiation of Endometrial and Vaginal Cancers-A Case Series and Review of Literature," Radiotherapy \& Oncology, Vol. 56, No. 1, 2000, pp. 23-28. http://dx.doi.org/10.1016/S0167-8140(00)00178-X

[26] E. Tsiridis, N. Upadhyay and P. V. Giannoudis, "Sacral Insufficiency Fractures: Current Concepts of Management," Osteoporosis International, Vol. 17, No. 12, 2006, pp. 1716-1725. http://dx.doi.org/10.1007/s00198-006-0175-1

[27] A. Moreno, J. Clemente, C. Crespo, et al., "Pelvic Insufficiency Fractures in Patients with Pelvic Irradiation," International Journal of Radiation Oncology * Biology * Physics, Vol. 44, No. 1, 1999, pp. 61-66. http://dx.doi.org/10.1016/S0360-3016(98)00534-3

[28] J. Lin, E. Lachmann and W. Nagler, "Sacral Insufficiency Fractures: A Report of Two Cases and a Review of the Literature," Journal of Women's Health and Gender 
Based Medicine, Vol. 10, No. 7, 2001, pp. 699-705. http://dx.doi.org/10.1089/15246090152563588

[29] J. Kamysz and M. Rechitsky, "Pubic Bone Cement Osteoplasty for Pubic Insufficiency Fractures," Journal of Vascular and Interventional Radiology, Vol. 19, No. 9, 2008, pp. 1386-1389.

http://dx.doi.org/10.1016/j.jvir.2008.05.026

[30] J. Kamsyz, "Percutaneous Repair of a Nonunion Pubic Ramus Fracture Using a Metallic Stent Scaffold and Cement Osteoplasty," Journal of Vascular and Interventional Radiology, Vol. 21, No. 8, 2010, pp. 1313-1316. http://dx.doi.org/10.1016/j.jvir.2010.04.017

[31] D. Mears and J. Velyvis, "In Situ Fixation of Pelvic Nonunions Following Pathologic and Insufficiency Fractures," Journal of Bone and Joint Surgery, Vol. 84, No. 5,
2002, pp. 721-728.

[32] I. Papanastassiou, M. Setzer, M. Eleraky, et al., "Minimally Invasive Sacroiliac Fixation in Oncologic Patients with Sacral Insufficiency Fractures Using a Fluoroscopybased Navigation System," Journal of Spinal Disorders \& Techniques, Vol. 24, No. 2, 2011, pp. 76-82. http://dx.doi.org/10.1097/BSD.0b013e3181df8e6b

[33] D. Wahnert, M. Raschke and T. Fuchs, "Cement Augmentation of the Navigated Iliosacral Screw in the Treatment of Insufficiency Fractures of the Sacrum. A New Method Using Modified Implants," International Orthopedics, Vol. 37, No. 6, 2013, pp. 1147-1150. http://dx.doi.org/10.1007/s00264-013-1875-8 